\title{
Nonnative Accent Discrimination with Words and Sentences
}

\author{
Eriko Atagi Tessa Bent \\ Department of Speech and Hearing Sciences, Indiana University, \\ Bloomington, IN, USA
}

\begin{abstract}
Background: Accent detection studies have shown native listeners to be highly sensitive to the presence of nonnative accents. This study examined the robustness of this sensitivity. Methods: We evaluated listeners' accent discrimination performance when presented with a stimulus set consisting of multiple nonnative accents, as well as words and sentences that were unique in each trial. Listeners heard pairs of talkers reading the same word or sentence and indicated whether the talkers' native languages were the same or different. Talkers included two native talkers and six nonnative talkers from three native language backgrounds. Results: Listeners were highly sensitive to the difference between native and nonnative accents, confirming earlier findings, but were much less sensitive to the difference between two nonnative accents. Furthermore, while stimulus length affected listeners' sensitivity to the difference between native and nonnative accents, this factor had a minimal effect on their sensitivity to the difference between two nonnative accents. Conclusion: The findings suggest that task and stimulus characteristics have a significant effect on the degree of sensitivity to nonnative accents.
\end{abstract}

(C) 2017 S. Karger AG, Basel

\section{Introduction}

A nonnative accent can be generally defined as speech that systematically diverges from native speech due to interference from the phonological and acoustic-phonetic characteristics of a talker's native language. The specific characteristics of a nonnative accent will thus be shared across talkers from the same native language background and vary widely across different native language backgrounds (e.g., Flege et al., 1997; Flege and Fletcher, 1992). Other characteristics, such as a slower speech rate relative to native talkers, are shared across nonnative talkers regardless of native language background (Munro and Derwing, 1998, 2001). Nonnative accents can also vary substantially across talkers with the same native language background, due to reasons such as differences in degrees of target language acquisition - i.e., proficiency (e.g.,

\begin{tabular}{l}
\hline KARGER \\
$\begin{array}{l}\text { E-Mail karger@karger.com } 2017 \text { S. Karger AG, Basel } \\
\text { www.karger.com/pho }\end{array}$
\end{tabular}

\footnotetext{
Eriko Atagi

Department of Communication Sciences and Disorders

Northeastern University

Boston, MA 02115 (USA)

E-Mail e.atagi@northeastern.edu
} 
Flege, 1988; Flege et al., 1995). Despite the numerous ways in which nonnative speech can vary both across accents and across talkers, native listeners consistently discriminate nonnative from native accents, even in very short stimuli such as a monosyllabic word or a single phonetic segment (Flege, 1984; Park, 2013). Native listeners can even detect a nonnative accent when presented with the speech of nonnative talkers who acquired their second language during childhood and are highly proficient (Park, 2013; Flege and Fletcher, 1992).

Although accent detection studies have established that native listeners are quite sensitive to the presence of a nonnative accent (Flege, 1984; Park, 2013), the specific stimuli implemented in these studies may have substantially influenced listeners' sensitivity to nonnative accents. One notable methodological characteristic of these accent detection studies is the number of nonnative accents with which the listeners were presented. Specifically, these accent detection studies only presented listeners with nonnative talkers from one native language background to be contrasted from native talkers (Munro et al., 2010, experiment 3, in which Cantonese and Czech talkers were blocked by accent). Accent perception studies that contrasted native talkers with nonnative talkers from multiple native language backgrounds have utilized accent categorization (Vieru et al., 2011) and free classification (Atagi and Bent, 2013; McCullough and Clopper, 2016) tasks. In a seven-alternative forced-choice accent categorization task in French, Vieru et al. (2011) found that native listeners were nearly perfect at identifying the native French talkers (96\% accuracy); their overall identification rates for the six nonnative accent categories, in contrast, were significantly lower (54\%). Although these categorization results appear to indicate that listeners were clearly distinguishing native talkers from nonnative talkers, these listeners also misidentified some nonnative talkers as being native speakers of French - for example, native Arabic talkers were misidentified at a relatively high rate of $10 \%$. Similar results have been reported in two recent accent classification studies that presented their listeners with native talkers and nonnative talkers from six (Atagi and Bent, 2013) and four (McCullough and Clopper, 2016) language backgrounds. While both accent classification studies found native listeners to be highly accurate at grouping native talkers together, they also found that listeners classified some nonnative talkers with native talkers. The inclusion of multiple nonnative accents in the stimulus set in these accent categorization and free classification studies could have decreased the listeners' ability to discriminate between native and nonnative accents.

Ratings of foreign-accent strength have also shown that native listeners are quite sensitive not only to the presence of a foreign accent, but also to gradient foreign accent strength with native talkers consistently rated at one end of the spectrum (i.e., no foreign accent; Flege et al., 1995; Munro and Derwing, 1995a, b). Moreover, an effect of stimulus set has been demonstrated with a foreign-accent strength rating task: listeners rated nonnative talkers as more strongly accented when the proportion of native talkers included in the stimulus set increased (Flege and Fletcher, 1992). Therefore, the number of nonnative accents and the range of accent strengths in the free classification studies may have resulted in a less clear differentiation between native and nonnative talkers than in the accent detection studies. The range of variability across stimuli whether due to the presence of native talkers in the stimulus set or the number of different foreign accents included in the stimulus set as discussed above - may affect the perceptual salience of nonnative accent characteristics for listeners (Atagi and Bent, 2013). 
Accent categorization and free classification tasks can also provide insight into native listeners' ability to discriminate among different nonnative accents. Native listeners are able to categorize nonnative accents by native language background at above-chance levels (Atagi and Bent, 2015; Vieru et al., 2011). Native listeners also organize their classification along multiple dimensions that include both sociolinguistic characteristics (i.e., accent strength and native language background; Atagi and Bent, 2013) and acoustic-phonetic characteristics (e.g., voice onset time and vowel formant features; McCullough and Clopper, 2016). These results suggest that native listeners' perceptual sensitivity to nonnative accent characteristics is not restricted to a binary distinction between native and nonnative accents. Categorization and classification tasks, however, come with some methodological limitations. In an accent categorization task, listeners are provided with a set of preselected categories and thus are restricted in their response options. Although the free classification task presents a good alternative that allows listeners to group talkers by perceptual similarity without experimenterconstrained categories, its downside is that listeners do not need to make every pairwise comparison possible among the stimuli. Furthermore, the free classification task forces listeners to create boundaries within a continuous perceptual similarity space. As a result, when the listener judges $\mathrm{A}$ to be similar to $\mathrm{B}$ and $\mathrm{B}$ to be similar to $\mathrm{C}$, but $\mathrm{A}$ and $\mathrm{C}$ to be dissimilar, the listener must decide which two to group together ( $\mathrm{A}$ and $\mathrm{B}$, or $\mathrm{B}$ and $\mathrm{C}$ ). Such perceptual decisions could result in exaggerations of perceived similarities and/or differences between accents. No study has investigated whether native listeners are able to simply discriminate between pairs of speech stimuli by their accents.

In addition to limitations imposed by the experimental task and the number of accents included in the stimulus set, another factor that could influence listeners' perception of nonnative accents is stimulus length. Some of the nonnative accent perception studies have focused on listeners' abilities to recognize nonnative acousticphonetic features at the segmental or single-word level (Flege, 1984; McCullough and Clopper, 2016; Park, 2013). Longer utterances such as sentences, however, are not only more similar to real-life experiences with spoken language, but also provide listeners with additional acoustic-phonetic information. Compared to a segment or single word, sentences contain a greater number of phonemes and phoneme-to-phoneme transitions, providing more opportunities for acoustic-phonetic, between-accent differences to arise. Sentences also contain various prosodic cues to a nonnative accent that a monosyllabic word or a single segment would not (Holm, 2008; Jilka, 2000; Munro et al., 2010). For example, native listeners presented with backwards (content-masked) nonnative speech can still distinguish between native and nonnative talkers at abovechance levels (Munro et al., 2010). Furthermore, even when some of the prosodic information (e.g., pitch variation, temporal integrity) is removed or disrupted, listeners' accent detection remains above chance, although sensitivity is significantly reduced (Munro et al., 2010).

Another characteristic of previous accent detection, rating, and classification studies is the number of different linguistic stimuli tested in these studies. To identify specific acoustic-phonetic properties to which native listeners are sensitive (e.g., voice onset time), many previous studies repeatedly presented listeners with the same word or syllable, as produced by multiple talkers (e.g., Flege, 1984; Flege and Fletcher, 1992; Park, 2013). The multiple repetition of the same word or syllable may have allowed listeners to tune in to very detailed, specific speech features. The generalizability of such heightened perceptual sensitivity across a variety of utterances requires further investigation. 
Recent speech perception and encoding frameworks have suggested that listeners' ability to cognitively represent sociolinguistic information, such as the talker's accent or dialect, is as central to spoken language comprehension as the ability to represent linguistic information (Sumner et al., 2014). Further, the listeners' ability to construct a systematic representation of across-talker and across-group speech variability plays a central role in achieving robust speech recognition despite the lack of invariance in speech (Kleinschmidt and Jaeger, 2015). The use of such sociolinguistic information during nonnative speech processing may be relevant to the listeners' ability to phonetically adapt to nonnative speech and subsequently improve the intelligibility of novel nonnative talkers. Specifically, adaptation and generalization to novel nonnative talkers have been observed both after exposure to talkers whose native language background was the same as that of the novel talker (accent-specific generalization; Bradlow and Bent, 2008), as well as after exposure to nonnative speech generally, where intelligibility on a novel accent improved after exposure to talkers with a variety of accents (accent-independent generalization; Baese-Berk et al., 2013). Accent-independent generalization suggests that listeners perceive similarities across different nonnative accents. Moreover, these results indicate that native listeners' cognitive representation of nonnative speech variability is organized within a multidimensional, acousticphonetic similarity space. Whether native listeners can simultaneously recognize the similarities within a nonnative accent background as well as the differences across nonnative accent backgrounds, however, has received relatively little attention, other than in the two recent free classification studies (Atagi and Bent, 2013; McCullough and Clopper, 2016).

It remains an empirical question whether native listeners' heightened sensitivity to the presence of a nonnative accent may be limited to the comparison between a native accent and a nonnative accent. Furthermore, listeners' sensitivity to differences between native and nonnative accents could have been overestimated in previous accent detection studies, due to the limited range of stimuli with which listeners were presented (Flege, 1984; Munro et al., 2010; Park, 2013). The present study therefore examined the native listeners' sensitivity to nonnative accents using accent discrimination tasks. Specifically, experiment 1 implemented an accent discrimination task with one native and three nonnative accents, using a variety of sentence-length and monosyllabic word-length stimuli. Further, to determine whether the presence of a native accent in the stimulus set affected the listeners' perceptual sensitivity to nonnative accents, experiment 2 implemented the same task and stimuli without the nativeaccented stimuli. The present study was conducted with the following goals: (1) test listeners' sensitivity to the native-nonnative distinction when they are presented with three different nonnative accents, rather than one nonnative accent category; (2) investigate the extent to which stimulus length modulates listeners' perceptual sensitivity to nonnative accent characteristics, and (3) examine whether listeners can discriminate between different nonnative accents.

\section{Experiment 1}

Methods

Listeners

Fifty-six monolingual speakers of American English were recruited from the Indiana University campus. One listener was excluded for not following instructions, one did not pass the hearing 
Table 1. Baseline degrees of accent for the talkers included in the present study

\begin{tabular}{ll}
\hline Talker & Degree of accent \\
\hline English1 & 1.07 \\
English2 & 1.14 \\
German1 & 2.97 \\
Mandarin1 & 4.36 \\
German2 & 4.80 \\
Mandarin2 & 4.84 \\
Korean1 & 5.15 \\
Korean2 & 5.45 \\
\hline
\end{tabular}

Baseline measures were collected for the Hoosier database (Atagi and Bent, 2011). The overall degree of foreign accent for sentences was rated on a scale of $1=$ no foreign accent to $9=$ very strong foreign accent.

screening, one had extensive and recent experience living abroad, and two were excluded because they reported having family members who regularly spoke a non-English language in their homes. The remaining 51 adult monolingual native speakers of American English (30 females and 21 males) were included in the analyses. Their mean age was 21.2 years with a range of $18-35$ years. All but one of the listeners reported being born and raised in the USA. One listener did not report his residential history; however, he reported having no experience of studying or living abroad. Eight of the listeners included in the analyses had continuously studied and lived abroad for 1-5 months. Though none of these listeners reported being conversationally proficient in any language other than English, 49 reported having studied 1-3 of the following spoken languages: Spanish $(n=38)$, French $(n=10)$, German $(n=$ 5), Italian $(n=3)$, Japanese $(n=3)$, Mandarin $(n=2)$, Korean, and Twi $(n=1$ for each). On average, these listeners had 4.2 years of total foreign language study (range: $0-10$ years; excludes one listener who did not report the durations of his study of two foreign languages). All listeners included in the analyses had normal hearing thresholds of $\leq 20 \mathrm{~dB} \mathrm{HL}$ at 250, 500, 1,000, 2,000, 4,000, and 8,000 Hz, and reported no current speech or hearing impairment. Furthermore, they did not report any substantial experience interacting with nonnative speakers (e.g., immediate family members, partners, very close friends) from the three included native language backgrounds (German, Mandarin, Korean). They also reported limited to no instruction in linguistics, speech and hearing sciences, or any other related fields. Listeners were paid for their participation.

Stimuli: Talkers

Stimuli for the present study were selected from the Hoosier Database of Native and Nonnative Speech for Children (Atagi and Bent, 2013; Bent, 2014). This database contains recordings of words, sentences, and paragraphs that are lexically and syntactically at the appropriate level for use with children. At the time of recording, all of the nonnative talkers had spent four years or less in the USA or any other English-speaking country, and were only proficient in their native language and English (as a second language).

The talkers selected for use in this experiment were six nonnative talkers from three native language backgrounds and two native talkers (two talkers per native language background). All talkers were female. The native language backgrounds of these talkers were German (Upper Saxon dialect of east-central Germany), Mandarin (Beijing dialect), Korean (Seoul dialect), and American (Midland dialect). The six nonnative talkers were selected for this experiment based on their degrees of foreign accent. According to the baseline measurements collected for the database (Atagi and Bent, 2011), these six nonnative talkers' overall degrees of foreign accent span the range from mildly accented to moderately accented (Table 1). Degree of accent, considered to be a subjective measure (Derwing and Munro, 1997; Munro and Derwing, 1995a, b), was measured based on three representative sentences on a 9-point scale, where $1=$ no foreign accent and $9=$ very strong foreign accent (Atagi and Bent, 2011). 
Stimuli: Items

The stimuli consisted of 192 words and 192 sentences read by the eight talkers. The 192 words used in the experiment were all monosyllabic words. The 192 sentences selected from the Hoosier database (Atagi and Bent, 2013) consisted of sentences in the Bamford-Kowal-Bench Standard Sentence Test (Bamford and Wilson, 1979) and the Hearing in Noise Test - Children's Version (Nilsson et al., 1996), which are short sentences that are on average five words long and generally contain three or four content words (e.g., "The front yard is pretty"). Each item (word/sentence) was played in one trial only.

Procedure

Listeners first completed a hearing screening and a language background questionnaire, followed by two accent discrimination tasks: words and sentences. The order of the two accent discrimination tasks was counterbalanced across listeners. The experiment was written in GNU Octave (http://www.gnu.org/software/octave/) and was presented to listeners on a Mac minicomputer using Psychtoolbox (Brainard, 1997; Kleiner et al., 2007). Listeners were seated in a sound-treated booth, and stimuli were presented binaurally in quiet at approximately $68 \mathrm{~dB}$ SPL through Sennheiser HD 280 pro headphones.

In each accent discrimination task, listeners were presented with 192 trials. Each trial consisted of two presentations of the same word or sentence. There were three pairing types: (1) same talker same accent, (2) different talker - same accent, and (3) different talker - different accent. Listeners were instructed to "decide whether the two recordings of a word/sentence were spoken in the same accent or different accent." In addition to instructions given on the computer screen, the experimenter gave verbal instructions and ensured that listeners understood that "accent" referred only to nonnative accents (i.e., not regional dialect differences or any other native varieties).

In each trial, listeners first saw a center cross for $500 \mathrm{~ms}$ that indicated the start of a new trial, followed by a visual presentation of the word or sentence in the middle of the computer screen. The stimulus item was visually presented to minimize possible effects of intelligibility. After a 1-s delay, listeners heard the two auditory presentations of the item with an interstimulus interval of $500 \mathrm{~ms}$, which was kept short to minimize memory decay, but long enough for a clearly perceptible pause between the two items. Immediately after each pair of recordings had finished playing, the prompt, "SAME ACCENT or DIFFERENT ACCENT?" appeared on the computer screen (Fig. 1). Listeners pressed "s" for same and "d" for different on the keyboard to indicate their response. The next trial started automatically following the listener's response. When the same talker was presented in a trial, the two presentations were different tokens. Talker assignment for each item (word/sentence) as well as the order in which items were presented were randomized across listeners.

The number of same and different trials was balanced. The "same" trials consisted of sametalker pairs and pairs of different talkers who had the same accent background. To achieve a balanced number of same and different trials, same-accent pairs were presented six times per ordered talker pair, whereas different-accent pairs were presented twice per ordered talker pair. The same and different trials were further subdivided by multiple types of accent pairings, in which trials were separated by whether the pair included a native talker at the first level of subdivision, and the second level of subdivisions was separated by the specific accent pairs (Fig. 2).

\section{Results}

To evaluate the listeners' overall accent discrimination performance and accent discrimination given different types of accent pairs, the listeners' sensitivity was calculated using signal detection theory analysis (Macmillan and Creelman, 2005) as is applied to a change detection task. Trials in which talkers 1 and 2 have different accents (i.e., "different" trials) count as the signal trials; trials in which talkers 1 and 2 have the same accent (i.e., "same" trials) count as the no-signal trials. The listeners' performance on this accent discrimination task is defined as their sensitivity to a change in accent between talker 1 and talker 2, measured in $d^{\prime}$. A higher $d^{\prime}$ indicates better accent discrimination accuracy and higher sensitivity. 


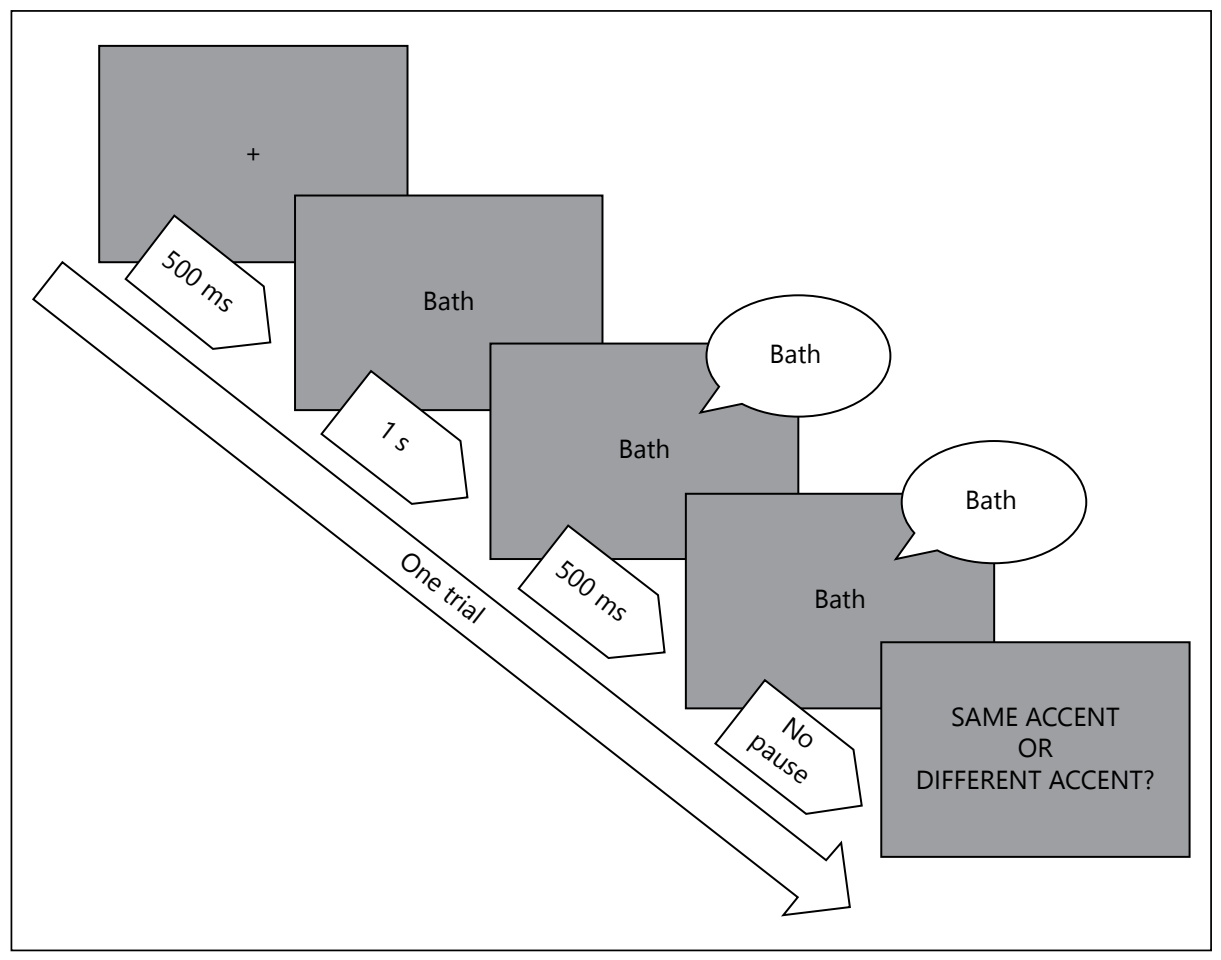

Fig. 1. Experiment procedure.

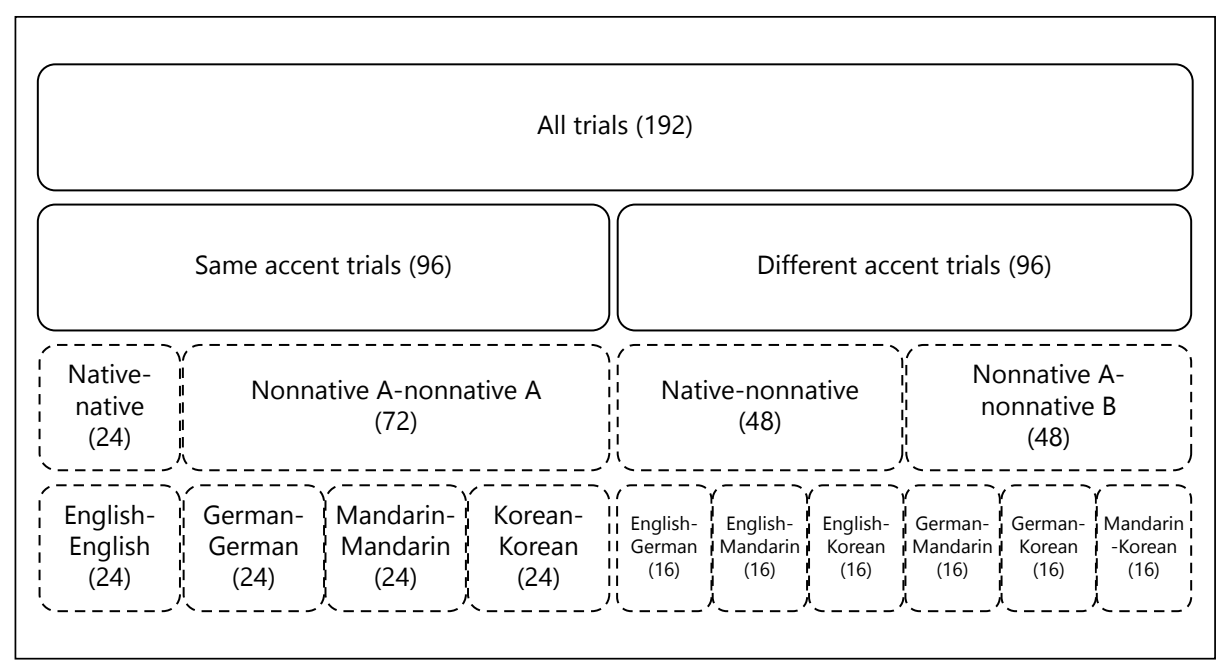

Fig. 2. Breakdown of the 192 accent discrimination trials by pairing type. Numbers in parentheses indicate the total number of trials per accent pairing type. 


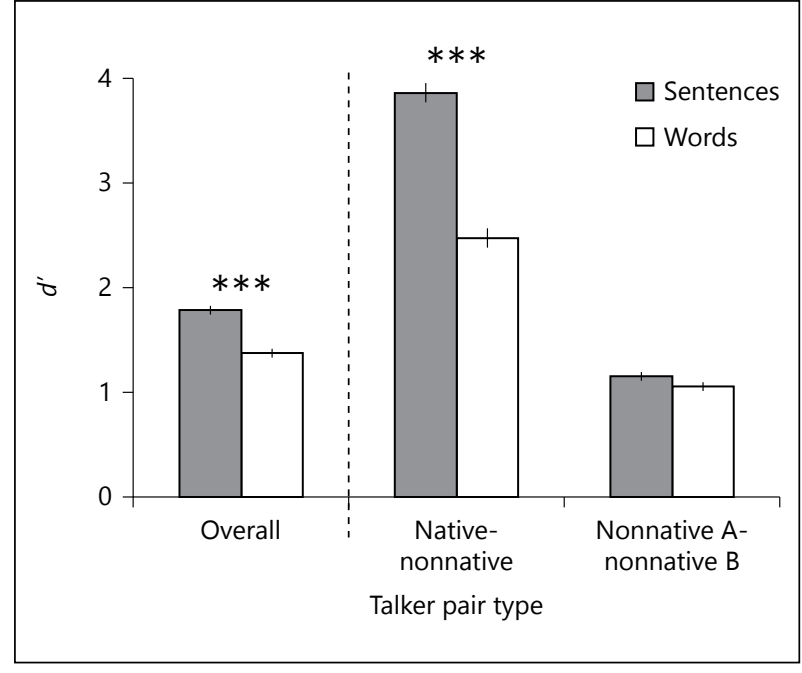

Fig. 3. Listeners' sensitivity to accent change. Error bars indicate standard error of the mean. Asterisks indicate significant differences between the sentence and word conditions $(* * * p<0.0001)$.

Table 2. Specific accent pair trials used to calculate hits and false alarms for native-included and nonnative-only accent pair types

\begin{tabular}{lll}
\hline Accent pair type & Hit trials & False alarm trials \\
\hline Native-included & English-German, & English-English \\
& English-Mandarin, & \\
English-Korean & \\
\hline Nonnative-only & German-Mandarin, & German-German, \\
& German-Korean, & Mandarin-Mandarin, \\
& Mandarin-Korean & Korean-Korean
\end{tabular}

Overall Performance: Sentence Condition versus Word Condition

To examine differences in listeners' overall accent discrimination performance in the sentence and word conditions, each listener's overall $d^{\prime}$ (sensitivity across all trials) was calculated separately for the word and sentence conditions. The leftmost pair of bars in Figure 3 shows that the overall $d^{\prime}$ was higher in the sentence condition than in the word condition. A two-tailed paired $t$ test confirmed that listeners' overall sensitivity in the sentence condition (mean $=1.78, \mathrm{SD}=0.30$ ) was significantly higher than in the word condition $($ mean $=1.37, \mathrm{SD}=0.29), t(50)=9.4, p<0.0001$.

Native-Included Pairs versus Nonnative-Only Pairs

The listeners' sensitivity to accent differences was compared for talker pairs that included a native talker (i.e., native-native or native-nonnative) and for pairs with two nonnative talkers by calculating the $d^{\prime}$ for native-included pairs and nonnative-only pairs. These $d$ ' values were calculated as the proportion of "different" responses in the hit and false alarm trials as indicated in Table 2 . Both the native-included and nonnative-only $d^{\prime}$ values were calculated separately (Fig. 3) for the sentence condition (native-included: 
Table 3. Accent pair trials used to calculate hits and false alarms for specific accent pairs

\begin{tabular}{lll}
\hline Accent pair type & $\begin{array}{l}\text { Specific accent pair } \\
\text { (hit trials) }\end{array}$ & False alarm trials \\
\hline Native-nonnative & English-German & English-English, German-German \\
& English-Mandarin & English-English, Mandarin-Mandarin \\
& English-Korean & English-English, Korean-Korean \\
\hline Nonnative A-nonnative B & German-Mandarin & German-German, Mandarin-Mandarin \\
& German-Korean & German-German, Korean-Korean \\
& Mandarin-Korean & Mandarin-Mandarin, Korean-Korean \\
\hline
\end{tabular}

mean $=3.86, \mathrm{SD}=0.65$; nonnative-only: mean $=1.15, \mathrm{SD}=0.32)$ and the word condition (native-included: mean $=2.47, \mathrm{SD}=0.65$; nonnative-only: mean $=1.05, \mathrm{SD}=0.29$ ).

As seen in Figure 3, listeners were more sensitive to changes in accent when the stimuli were sentences rather than words, and were also more sensitive to a change between a native-nonnative pair than between a pair of two nonnative accents. A $2 \times 2$ repeated-measures analysis of variance was performed with condition (sentence or word) and pair type (native-included or nonnative-only), which confirmed significant main effects for both condition, $F(1,150)=249.2, p<0.0001$, and pair type, $F(1,150)=947.8, p<0.0001$. The interaction between condition and pair type was also significant, $F(1,150)=107.5, p<0.0001$.

Post hoc two-tailed paired $t$ tests with Bonferroni correction for each pair type were conducted to further examine the interaction effect $(\alpha=0.025)$. For native-nonnative pairings, the listeners' overall sensitivity in the sentence condition (mean $=3.86$, $\mathrm{SD}=0.65$ ) was significantly higher than in the word condition (mean $=2.47, \mathrm{SD}=$ $0.65)$, with $t(50)=12.8$ and $p<0.0001$. However, the listeners' sensitivity to change between 2 nonnative accents did not differ significantly between the sentence (mean $=$ $1.15, \mathrm{SD}=0.32$ ) and word (mean $=1.05, \mathrm{SD}=0.29)$ conditions, $t(50)=1.9$, ns. These results indicate that sentences only led to higher accent sensitivity for pairs in which a native talker was included. To examine the interaction between stimuli type (sentence vs. word) and accent pair in more detail, analyses of listeners' sensitivity to specific accent pairs were conducted.

\section{Specific Accent Pairs: Sensitivity to Native-Nonnative Accent Changes}

The $d^{\prime}$ for specific native-nonnative accent pairs were calculated by defining hits and false alarms as proportion of "different" responses in the trials indicated in the top half of Table 3. The accent-specific $d^{\prime}$ was calculated separately for the sentence and word conditions, yielding six sensitivity measures: English-German (sentence: mean $=2.69, \mathrm{SD}=0.64$; word: mean $=1.51, \mathrm{SD}=0.49$ ), English-Mandarin (sentence: mean $=2.90, \mathrm{SD}=0.72$; word: mean $=2.16, \mathrm{SD}=0.72$ ), and English-Korean (sentence: mean $=3.31, \mathrm{SD}=0.79$; word: mean $=1.86, \mathrm{SD}=0.62$ ).

The listeners' sensitivity was higher with sentences than with words across all native-nonnative accent pairs (Fig. 4, left side). Three paired $t$ tests for each accent pair were conducted with Bonferroni correction $(\alpha=0.017)$, which confirmed that listeners were significantly more sensitive to the accent differences when listening to 


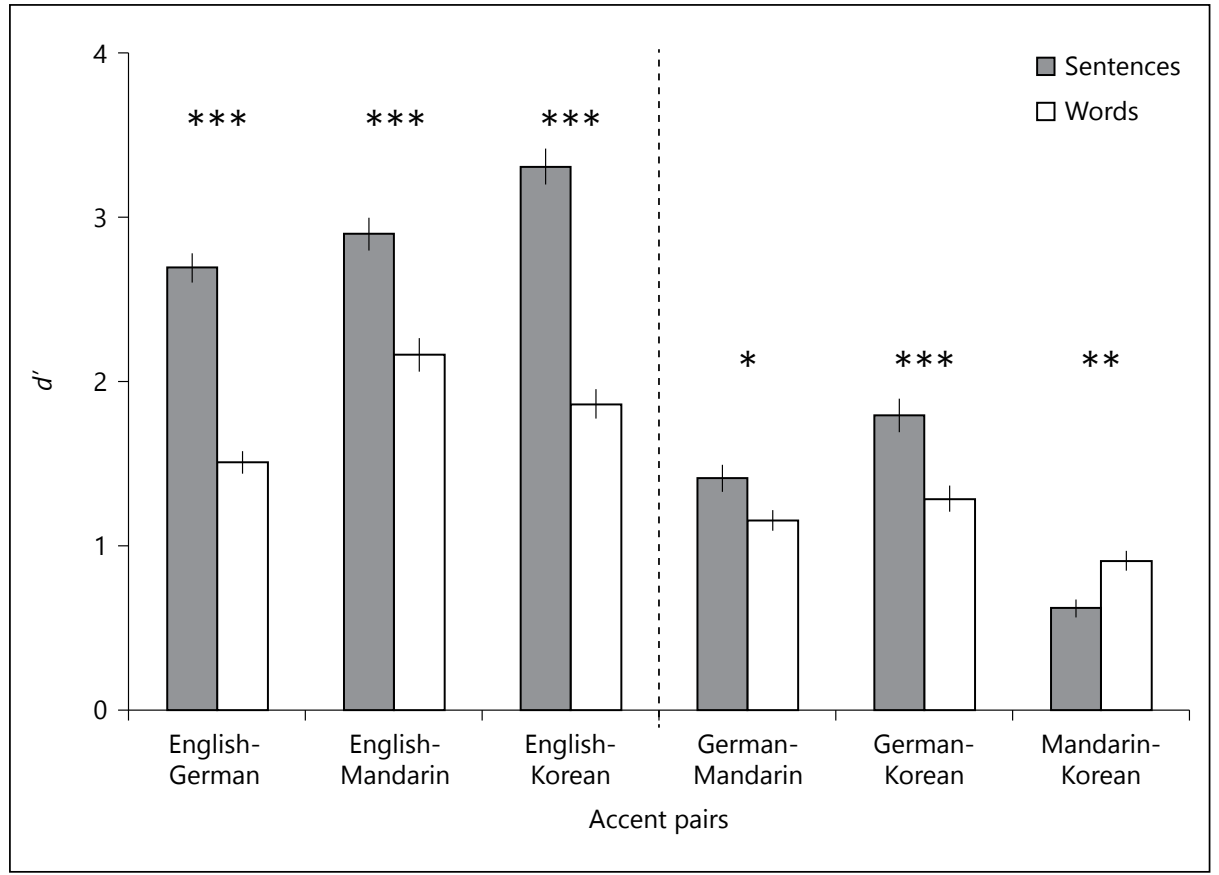

Fig. 4. Listeners' sensitivity to specific native-nonnative and nonnative A-nonnative B accent changes. Error bars indicate standard error of the mean. Asterisks indicate significant differences between the sentence and word conditions $(* p<0.01, * * p<0.001, * * * p<0.0001)$.

sentence-length stimuli than word-length stimuli for all three pairs: English-German, $t(50)=11.7, p<0.0001$; English-Mandarin, $t(50)=6.6, p<0.0001$; and EnglishKorean, $t(50)=12.6, p<0.0001$.

Specific Accent Pairs: Sensitivity to Accent Changes between Two Nonnative Accents

The $d^{\prime}$ for specific nonnative-nonnative accent pairs was calculated by defining hits and false alarms as proportion of "different" responses in the trials indicated in the bottom half of Table 3 . The accent-specific $d^{\prime}$ was again calculated separately for the sentence and word conditions, yielding six sensitivity measures: German-Mandarin (sentence: mean $=1.41, \mathrm{SD}=0.59$; word: mean $=1.15, \mathrm{SD}=0.45$ ), German-Korean (sentence: mean $=1.79, \mathrm{SD}=0.74$; word: mean $=1.29$, $\mathrm{SD}=0.57$ ), and MandarinKorean (sentence: mean $=0.62, \mathrm{SD}=0.40$; word: mean $=0.91, \mathrm{SD}=0.44$ ).

The listeners' sensitivity varied depending on the presentation condition and the accent pair (Fig. 4, right side). Listeners showed the expected greater sensitivity to sentence-length stimuli than to word-length stimuli for German-Mandarin and GermanKorean accent pairs; however, for the Mandarin-Korean accent pair, listeners showed greater sensitivity to word-length than sentence-length stimuli. Paired $t$ tests for each accent pair with Bonferroni correction $(\alpha=0.017)$ confirmed these differences in sensitivity across stimulus length conditions: German-Mandarin, $t(50)=2.6, p<0.01$; German-Korean, $t(50)=5.0, p<0.0001$; Mandarin-Korean, $t(50)=-4.0, p<0.001$. 


\section{Conclusion}

Overall, listeners were more sensitive to the difference in accent when presented with sentence-length stimuli than word-length stimuli. In contrast, listeners' overall perceptual sensitivity to accent differences in nonnative-nonnative pairs was not significantly different between sentences and words. Without examining the specific accent pairs, the overall effect of stimulus length would have appeared to be driven by the trials when listeners were presented with a native-nonnative pair. Further examination of the specific accent pairs, however, revealed that the Mandarin-Korean accent pair differed from all other accent pairs in that listeners were more sensitive to the change in accent when presented with words than sentences. Nevertheless, the listeners' level of sensitivity to the accent change between Mandarin and Korean accents was quite low with both the sentence and word-length stimuli; it is thus difficult to determine whether this opposite pattern observed for this accent pair represents a meaningful difference. Overall, listeners were not highly sensitive to differences between two nonnative accents, regardless of the stimulus condition. To investigate whether the inclusion of native talkers in the stimulus set caused listeners to have limited sensitivity to the differences between two nonnative accents (Flege and Fletcher, 1992), experiment 2 was conducted with a stimulus set that consisted of only nonnative talkers.

\section{Experiment 2}

In a nonnative accent rating task, Flege and Fletcher (1992) observed an effect of stimulus set - when a greater proportion of native talkers were included in the stimulus set, the perceived accent strength of the nonnative talkers increased. Experiment 2 thus investigates whether such stimulus set effects may have played a role in experiment 1. Specifically, listeners may have been less sensitive to differences between nonnative accents due to the much more perceptually salient differences between a native accent and a nonnative accent. The subtle differences between two nonnative accents may be less perceptually salient in the context of the much greater perceptual distance between a native accent and a nonnative accent. If the presence of native talkers decreased listeners' perceptual sensitivities to the differences among nonnative accents in experiment 1 , then a stimulus set that consists only of nonnative talkers may allow listeners in experiment 2 to detect between-accent differences with greater sensitivity.

\section{Methods}

Listeners

A new group of 54 monolingual speakers of American English was recruited from the Indiana University campus. One listener was excluded from the analyses because he was born and partially raised outside of the USA. The remaining 53 adult monolingual native speakers of American English ( 37 females and 16 males) had a mean age of 20.7 years with a range of 18-33 years. Four of the listeners had study abroad experiences of less than a year each. Though none of the listeners reported being conversationally proficient in any language other than English, 49 reported having studied 1-4 of the following spoken languages: Spanish $(n=37)$, German $(n=8)$, French $(n=7)$, Chinese $(n=3)$, Japanese $(n=2)$, Italian $(n=2)$, Korean, Greek, Swahili, Portuguese, and Arabic $(n=1$ for each). On average, these listeners had 6.0 years of total foreign language study (range: 0-25 years; excludes 1 listener who did not report the duration of his study of a foreign language). All listeners included in the analyses had normal hearing thresholds of $\leq 25 \mathrm{~dB} \mathrm{HL}$ at $250 \mathrm{~Hz}$, and $\leq 20 \mathrm{~dB}$ HL at $500,1,000,2,000$, 4,000 , and $8,000 \mathrm{~Hz}$, and reported no current speech or hearing impairment. Only three of the included listeners reported substantial experience interacting with nonnative speakers from the three included 


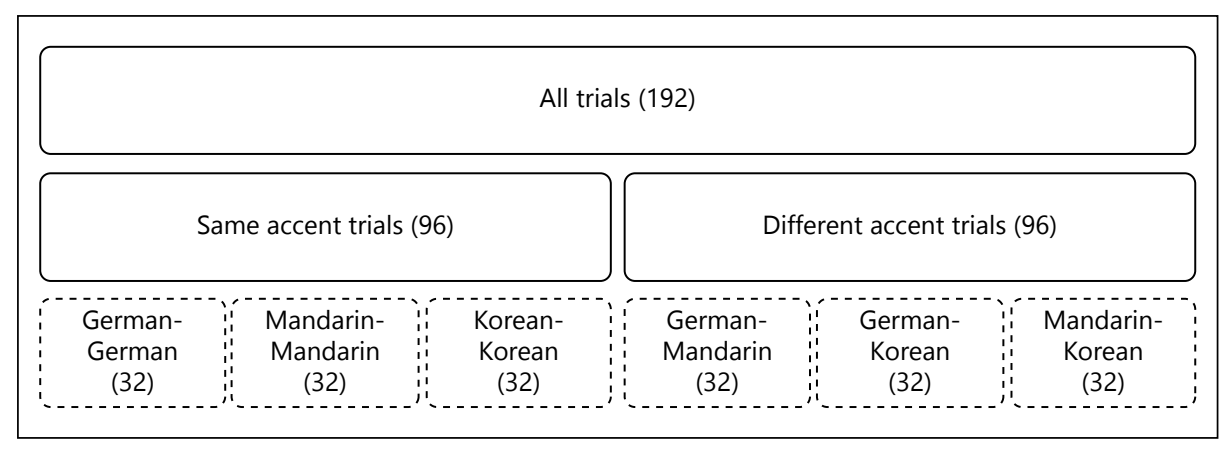

Fig. 5. Breakdown of the 192 accent discrimination trials by pairing type. Numbers in parentheses indicate the total number of trials per accent pairing type.

native language backgrounds; these three listeners reported tutoring English to nonnative speakers (specifically, "Korean students," "German and Chinese students," or "nonnative speakers from China and East Asia"). Listeners were paid for their participation.

Stimuli

The same six nonnative talkers used in experiment 1 were again used in experiment 2 . The same stimuli (192 sentences and 192 words) were used in experiment 2 as in experiment 1.

Procedure

The general procedure was the same as in experiment 1 . The number of trials in each accent pair is shown in Figure 5. To achieve a balanced number of same and different trials, same-accent pairs were presented eight times per ordered talker pair, whereas different-accent pairs were presented four times per ordered talker pair.

\section{Results}

Overall Performance: Sentence Condition versus Word Condition

As in experiment 1 , the overall $d^{\prime}$ for each listener was calculated across all trials, separately for the sentence condition (mean $=1.15, \mathrm{SD}=0.30)$ and the word condition (mean $=1.15, \mathrm{SD}=0.40)$. A two-tailed paired $t$ test confirmed that listeners' performance across these two conditions did not differ significantly, $t(52)=0.1$, ns (Fig. 6, left side).

\section{Specific Accent Pairs}

The $d^{\prime}$ for specific pairs of nonnative accents was calculated by defining hits and false alarms as proportion of "different" responses in the trials indicated in Table 4. The accent-specific $d^{\prime}$ was again calculated separately for the sentence and word conditions, yielding 6 sensitivity measures: German-Mandarin (sentence: mean $=1.45, \mathrm{SD}=$ 0.61 ; word: mean $=1.23, \mathrm{SD}=0.63)$, German-Korean $($ sentence: mean $=1.65, \mathrm{SD}=$ 0.67 ; word: mean $=1.45, \mathrm{SD}=0.76$ ), and Mandarin-Korean (sentence: mean $=0.76$, $\mathrm{SD}=0.41$; word: mean $=1.05, \mathrm{SD}=0.44$ ).

The listeners' sensitivity varied depending on the condition and the accent pair (Fig. 6, right side). To test the differences between stimulus conditions for each accent pair, 3 paired $t$ tests with Bonferroni correction $(\alpha=0.017)$ were performed. The German-Mandarin and German-Korean pairs did not yield significantly different $d^{\prime}$ between the sentence and word conditions: German-Mandarin,

\begin{tabular}{lll}
\hline 184 & $\begin{array}{l}\text { Phonetica 2017;74:173-191 } \\
\text { DOI: } 10.1159 / 000452956\end{array}$ & Atagi/Bent
\end{tabular}




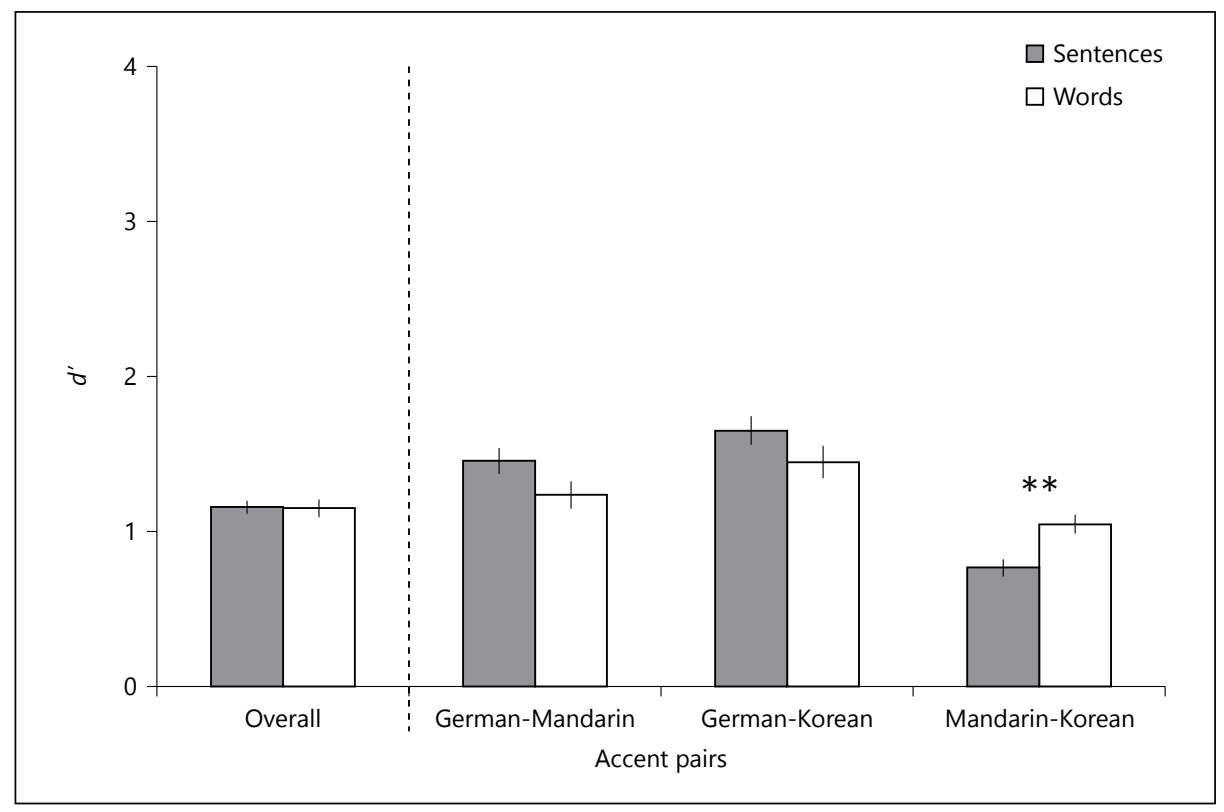

Fig. 6. Listeners' sensitivity overall and to specific accent changes. Error bars indicate standard error of the mean. Asterisks indicate significant differences between the sentence and word conditions $(* * p<0.001)$.

Table 4. Specific accent pair trials used to calculate hits and false alarms for native-included and nonnative-only accent pair types

\begin{tabular}{ll}
\hline Accent pair/hit trials & False alarm trials \\
\hline German-Mandarin & German-German, Mandarin-Mandarin \\
German-Korean & German-German, Korean-Korean \\
Mandarin-Korean & Mandarin-Mandarin, Korean-Korean \\
\hline
\end{tabular}

$t(52)=2.2$, ns; German-Korean, $t(52)=1.7$, ns. With the Mandarin-Korean pair, $d^{\prime}$ for words was higher than for sentences as was found in experiment $1, t(52)=-3.5$, $p<0.001$.

\section{Conclusion}

Similar to the results of experiment 1 , listeners were not highly sensitive to differences between two nonnative accents in experiment 2; the listeners' overall sensitivity to accent change did not differ significantly between sentence and word-length stimuli either. Unlike the results of experiment 1, however, listeners did not show a sentence advantage in discriminating between any of the nonnative accent pairs. The results for the Mandarin-Korean accent pair again indicated that listeners were more sensitive to the accent change when presented with words than sentences. 


\section{Discussion}

The present study examined native listeners' ability to discriminate between (1) native and nonnative accents, and (2) different nonnative accents. In contrast to prior nonnative accent detection studies which only employed a single nonnative accent (Flege, 1984; Park, 2013), the listeners' sensitivity to the native-nonnative distinction was examined with nonnative talkers from three different native language backgrounds. Further, the present study examined how stimulus length affected the native listeners' accent discrimination ability.

\section{Effect of Accent Pair Type on Accent Discrimination Sensitivity}

The listeners' sensitivity to the difference between a native accent and a nonnative accent was significantly higher than their sensitivity to the difference between two nonnative accents. However, listeners still demonstrated some sensitivity to the differences between all nonnative accent pairs ( $d^{\prime}$ values ranged from 0.62 for Mandarin-Korean to 1.79 for German-Korean with sentence-length stimuli, experiment 1), despite the relatively similar degrees of accent strength across the nonnative talkers. This result is consistent with the classification accuracy and perceptual similarities found in free classification studies of nonnative speech (Atagi and Bent, 2013; McCullough and Clopper, 2016). In both classification studies, native listeners were markedly better at grouping native talkers than nonnative talkers by native language background.

In experiment 2, listeners' discrimination of nonnative accents was examined when native talkers were not part of the stimulus set. Although a new group of listeners were recruited for experiment 2, the $d^{\prime}$ levels stayed remarkably consistent across the two experiments. These results indicate that although native listeners exhibited some ability to discriminate between two nonnative accents, their ability to discriminate between nonnative accents is much more limited than their sensitivity to the nativenonnative distinction.

The results of the present study, combined with those of prior accent classification and detection studies, suggest that the native listeners' ability to differentiate between native and nonnative accents may be a result of the listeners' acute recognition of deviation from native language norms. This sensitivity to divergence may be a result of listeners basing their judgments of nonnative-ness based on a mismatch to expected speech characteristics (Van Engen and Peelle, 2014). That is, rather than detecting particular acoustic-phonetic characteristics as being nonnative or indicative of a particular accent, listeners may be primarily attending to when particular characteristics do not meet their expectations of native speech. There are numerous possible phonetic characteristics that could cue nonnative speech. Significant attentional and cognitive resources would be required for listeners to hold detailed acoustic-phonetic representations of every nonnative cue in working memory. Instead, listeners may take note of phonetic cues that do not match their expected pattern of speech. Each piece of information that goes against the default expectation leads the listener towards deciding that the talker has a nonnative accent. This perceptual strategy would result in listeners performing with higher accuracy in their discrimination between native and nonnative accents than between two nonnative accents - consistent with the observed results of the present study.

When discriminating between two nonnative accents, the perceptual strategy of expecting native speech would be ineffective. To discriminate between two nonnative

Atagi/Bent 
accents, it would no longer be sufficient to judge both talkers as being nonnative. Instead, listeners must compare two speech stimuli that both contain unfamiliar acoustic-phonetic characteristics. The decision process involved with accurately discriminating between two nonnative accents thus requires listeners to pay close attention to differences in fine acoustic-phonetic details. Then, as in nonnative accent free classification tasks (Atagi and Bent, 2013; McCullough and Clopper, 2016), listeners must determine whether these acoustic-phonetic characteristics are similar enough to be judged as belonging to the same accent. Unlike in the free classification tasks, however, listeners in the present study only had one chance to hear the stimuli. This methodological restriction likely introduced a level of complexity to the task that the free classification tasks did not.

Differences in foreign-accent strengths across nonnative talkers also contribute to differences in the acoustic-phonetic characteristics of nonnative speech, which likely further complicated listeners' accent discrimination performance. In the many acoustic dimensions along which talkers could be evaluated (e.g., voice onset time, vowel formant characteristics; McCullough and Clopper, 2016), less accented nonnative talkers from different native language backgrounds could be more similar to one another than to more strongly accented nonnative talkers with whom a native language is shared. Such an effect of foreign-accent strength, for example, has been observed in a free classification task when listeners grouped native and nonnative talkers by perceived native language background: the perceptual distance between native French talkers who were less accented and the native English talkers was noticeably shorter than the distance to the other native French talkers with a stronger foreign accent (Atagi and Bent, 2013).

Although care was taken in the present study to select talkers who were as similar as possible in accent strength (i.e., previously rated as having mild-to-moderate degrees of accent), the native language backgrounds were graded in their average degrees of accent (Table 1). When presented with sentence-length stimuli, listeners' levels of sensitivity to specific native-nonnative differences appeared to be related to the nonnative talkers' degrees of accent: lowest sensitivity to the English-German accent pairing (German talkers were on average least accented), and highest sensitivity to the English-Korean accent pairing (Korean talkers were most accented). The order of sensitivity levels was different for the word condition (i.e., listeners were most sensitive to the English-Mandarin accent pairing); however, this difference in results may be due to the fact that the accent ratings were conducted with sentence-length stimuli (Atagi and Bent, 2011). Future study should examine whether native listeners' ability to discriminate between two nonnative accents would be more similar to their ability to discriminate between native and nonnative accents if the nonnative talkers were more strongly accented.

\section{Effect of Stimulus Length on Accent Discrimination Sensitivity}

The present study investigated the extent to which listeners' discrimination of native and nonnative accents was affected by stimulus length. Listeners' sensitivity to the distinction between native and nonnative accents was significantly higher for sentence-length stimuli than word-length stimuli. This finding parallels the observation across experiments in Flege (1984), where listeners' accent detection accuracy was higher in the experiments that used longer stimuli (i.e., a phrase or a CV word), than the experiments that used very short stimuli (i.e., a single phonetic segment). Also consistent with previous findings of accent detection studies (Flege, 1984; Park, 2013), 
listeners in the present study were highly sensitive to the difference between native and nonnative accents in short stimuli. If listeners utilize the listening strategy of expecting native speech as discussed above, in most cases, the presence of a nonnative accent can be detected relatively accurately with very little information. Longer stimuli, such as sentences, provide more opportunities to further confirm nonnative-ness and result in listeners being highly accurate in discriminating between native and nonnative accents.

In contrast to the results of the native-nonnative discrimination, the advantage of the sentence-length stimuli over word-length stimuli was minimal or did not exist for discriminating between two nonnative accents, especially with the Mandarin-Korean pair. Furthermore, the advantage of sentence-length stimuli over word-length stimuli that was observed when native talkers were included in the stimulus set (experiment 1) was not observed at all when native talkers were not included (experiment 2). This differing pattern of results between the two experiments suggests that stimulus set effects (e.g., distribution of accent types in an experiment; Flege and Fletcher, 1992) played a subtle role in shaping listeners' perception of nonnative speech. Specifically, listeners' perceptual sensitivity to differences between nonnative accents was shaped by an interaction between stimulus length and inclusion of native talkers in the stimulus set. The listeners' sensitivity to nonnative accent characteristics is thus not a simple function of the amount of speech signal available, particularly when comparing two nonnative accents.

One possible reason for this lack of benefit from sentence-length stimuli during the discrimination of two nonnative accents may be due to the working memory load incurred by the task of comparing two nonnative-accented sentences. While the additional acoustic-phonetic information in sentences could increase the opportunity for relevant feature detection, it could also increase the working memory load as listeners attempt to hold in memory detailed acoustic-phonetic characteristics of the stimuli. Furthermore, since most of the current listeners were inexperienced with the nonnative accents included in the current study, their relative unfamiliarity with detailed acoustic-phonetic characteristics of these nonnative accents may have resulted in the nonnative-accented stimuli imposing a greater working memory load compared to the native-accented stimuli. A greater working memory load for nonnative speech could cause listeners to fail in cognitively representing both talkers' nonnative acoustic-phonetic characteristics with enough detail for an accurate comparison. Thus, when comparing native talkers with nonnative talkers, the additional opportunities for accent feature detection afforded by the longer stimuli were able to positively affect listeners' accent discrimination performance. In contrast, when comparing two nonnative accents - particularly two accents that are perceptually similar (e.g., Mandarin-Korean) - listeners may not have been able to make use of all of the available perceptual information. The interaction observed in the present study between accent pair types (native-nonnative vs. nonnative-nonnative) and stimulus length (words vs. sentences) may be reflective of the differentially greater working memory load incurred by the task of comparing two nonnative accented sentences.

\section{Effect of Stimulus Variability on Accent Discrimination Sensitivity}

In past accent detection studies, listeners were presented with a very limited set of stimulus items and a single accent type across all talkers and trials (Flege, 1984; Munro et al., 2010; Park, 2013), allowing listeners to constrain their perceptual sensitivity to a very small subset of the acoustic-phonetic variability possible in accented speech. In the present study, in contrast, listeners heard unique stimulus items (words or sentences) 
in each trial; no item was repeated across trials within the experiment. Presented with multiple nonnative accents and many different stimulus items, listeners in the present study may have had a difficult time determining the relevant phonetic characteristics that could serve as cues for each of the three nonnative accents. Despite this challenging task design, listeners exhibited a high sensitivity to the difference between a native accent and a nonnative accent. This result points to the robustness with which native listeners are able to accurately discriminate native from nonnative talkers.

Although listeners demonstrated some sensitivity to the differences between pairs of nonnative accents, they exhibited a significantly lower sensitivity to the nonnativenonnative differences than to the native-nonnative differences. The present study did not empirically test to what degree the uniqueness of stimuli across all trials contributed to the difficulty of discriminating between nonnative accent pairs. It is therefore unclear whether the same listeners would have exhibited increased perceptual sensitivity for the differences between two nonnative accents if they had been repeatedly presented with one word or sentence. To further investigate task effects on listeners' perceptual sensitivity to acoustic-phonetic features of multiple nonnative accents, a future accent discrimination study could implement both conditions - one in which each trial is a new word/sentence, as in the present study, and one where the same word/sentence is presented across all trials.

\section{The Perceptual Similarity of Mandarin and Korean Accents}

Among all accent pairs tested in the present study, listeners showed the lowest sensitivity levels when discriminating between Mandarin- and Korean-accented English, regardless of the stimulus length condition. The difficulty with which native listeners discriminate between Mandarin and Korean accents is consistent with the free classification results reported by Atagi and Bent (2013) for sentences and McCullough and Clopper (2016) for disyllabic words. The only exception to this pattern was in McCullough and Clopper when listeners were presented with only a CV syllable that had been extracted from their disyllabic words. In this case, Korean- and Mandarinaccented speech did not cluster together in their analyses of listeners' free classification behavior. It should be noted that the talkers used in the present study were also included in the stimulus set used by Atagi and Bent (2013).

The perceptual similarity of Mandarin- and Korean-accented English is therefore a robust result seen across a variety of stimuli, as well as across different talkers. With nonmeaningful CV syllables as stimuli, however, this perceptual similarity appears to diminish (McCullough and Clopper, 2016). The monosyllabic, word-length stimuli used in the present study would fall between McCullough and Clopper's CV syllable and disyllabic words in stimulus length (i.e., number of phonemes), and resulted in the low discriminability between Mandarin and Korean accents that has been observed with longer, meaningful stimuli. It is possible that the use of nonmeaningful stimuli results in a slightly different perceptual process than when meaningful stimuli are used. When presented with nonmeaningful stimuli, listeners may become focused on acoustic-phonetic details, as no other linguistic cues are available. Future work should systematically examine the possible interaction between specific acoustic-phonetic cues and meaningfulness of the stimuli to further elucidate the perceptual mechanisms by which native listeners discriminate between nonnative accents.

Finally, as with all perceptual phenomena, listeners' experience shapes perceptual similarities of linguistic varieties. For example, listeners with limited experience 
with regional dialect varieties have been found to be worse at categorizing talkers by regional dialect (Clopper and Pisoni, 2004). The perceptual similarity of Mandarin and Korean accents for native listeners may partly be due to insufficient experience differentiating between nonnative speakers from different Asian backgrounds. Sociolinguistic experience is difficult to measure, however, and the present listeners showed no indication that they had greater experience with German accents. Nevertheless, Atagi and Bent (2016) observed that native speakers of Korean found Mandarin and Korean accents to be more perceptually differentiated than did native speakers of American English or native speakers of Spanish. Further work is necessary to understand the role of sociolinguistic experience in listeners' abilities to discriminate between nonnative accents.

\section{Conclusion}

Native listeners' ability to discriminate between native and nonnative accents is quite robust even when presented with unique items in each trial spoken in multiple nonnative accents. Although listeners performed with high sensitivity in discriminating between native and nonnative accents, their ability to discriminate between two nonnative accents was more limited. Further, while stimulus length (words vs. sentences) had an effect on listeners' sensitivity to the difference between native and nonnative accents, this effect was minimal when discriminating between two nonnative accents. These findings extend the results of prior foreign-accent detection studies that examined listeners' sensitivity to nonnative accents in very constrained acoustic-phonetic contexts (Flege, 1984; Munro et al., 2010; Park, 2013). The present results suggest that while native listeners are quite sensitive to the difference between native and nonnative accents, task and stimulus characteristics play a significant role in shaping the degree to which listeners can detect a nonnative accent.

\section{Acknowledgments}

This work was supported by NIH grant T32 DC00012 (to E.A.) from the National Institute on Deafness and Other Communication Disorders, T32 NS007292 from the National Institute of Neurological Disorders and Stroke (to E.A.), and the Research Partnership Award from the Hutton Honors College at Indiana University awarded to Emma Bonifield. The authors would also like to thank Emma Bonifield for collecting the data for experiment 2. A version of experiment 1 was presented at the 168th Meeting of the Acoustical Society of America, Indianapolis, IN, in October 2014.

\section{References}

Atagi E, Bent T (2011): Perceptual dimensions of nonnative speech; in Lee W-S, Zee E (eds): International Congress of Phonetic Sciences XVII. Hong Kong, City University of Hong Kong, pp 260-263.

Atagi E, Bent T (2013): Auditory free classification of nonnative speech. J Phon 41:509-519.

Atagi E, Bent T (2015): Relationship between listeners' nonnative speech recognition and categorization abilities. J Acoust Soc Am 137:EL44-EL50.

Atagi E, Bent T (2016): Auditory free classification of native and nonnative speech by nonnative listeners. Appl Psycholinguist 37:241-263.

Baese-Berk MM, Bradlow AR, Wright BA (2013): Accent-independent adaptation to foreign accented speech. J Acoust Soc Am 133:EL174-EL180. 
Bamford J, Wilson I (1979): Methodological considerations and practical aspects of the BKB sentence lists; in Bench J, Bamford J (eds): Speech-Hearing Tests and the Spoken Language of Hearing-Impaired Children. London, Academic Press, pp 148-187.

Bent T (2014): Children's perception of foreign-accented words. J Child Lang 41:1334-1355.

Bradlow AR, Bent T (2008): Perceptual adaptation to non-native speech. Cognition 106:707-729.

Brainard DH (1997): The psychophysics toolbox. Spatial Vision 10:433-436.

Clopper CG, Pisoni DB (2004): Homebodies and army brats: some effects of early linguistic experience and residential history on dialect categorization. Lang Var Change 16:31-48.

Derwing TM, Munro MJ (1997): Accent, intelligibility, and comprehensibility: evidence from four L1s. Stud Second Lang Acquis 19:1-16.

Flege JE (1984): The detection of French accent by American listeners. J Acoust Soc Am 76:692-707.

Flege JE (1988): Factors affecting degree of perceived foreign accent in English sentences. J Acoust Soc Am 84:7079.

Flege JE, Bohn OS, Jang S (1997): Effects of experience on non-native speakers' production and perception of English vowels. J Phon 25:437-470.

Flege JE, Fletcher KL (1992): Talker and listener effects on degree of perceived foreign accent. J Acoust Soc Am 91:370-389.

Flege JE, Munro MJ, MacKay IRA (1995): Factors affecting strength of perceived foreign accent in a second language. J Acoust Soc Am 97:3125-3134.

Holm S (2008): Intonational and Durational Contributions to the Perception of Foreign-Accented Norwegian; doctoral dissertation, Norwegian University of Science and Technology, Trondheim.

Jilka M (2000): The Contribution of Intonation to the Perception of Foreign Accent; doctoral dissertation, University of Stuttgart.

Kleiner M, Brainard D, Pelli D (2007): What's new in Psychtoolbox-3? Perception 36(ECVP abstract suppl):14.

Kleinschmidt DF, Jaeger TF (2015): Robust speech perception: recognize the familiar, generalize to the similar, and adapt to the novel. Psychol Rev 122:148.

Macmillan NA, Creelman CD (2005): Detection Theory: A User's Guide, ed 2. Mahwah, Erlbaum Associates.

McCullough EA, Clopper CG (2016): Perceptual subcategories within non-native English. J Phon 55:19-37.

Munro MJ, Derwing TM (1995a): Foreign accent, comprehensibility and intelligibility in the speech of 2nd language learners. Lang Learn 45:73-97.

Munro MJ, Derwing TM (1995b): Processing time, accent, and comprehensibility in the perception of native and foreign-accented speech. Lang Speech 38:289-306.

Munro MJ, Derwing TM (1998): The effects of speaking rate on listener evaluations of native and foreign-accented speech. Lang Learn 48:159-182.

Munro MJ, Derwing TM (2001): Modeling perceptions of the accentedness and comprehensibility of L2 speech: the role of speaking rate. Stud Second Lang Acquis 23:451-468.

Munro MJ, Derwing TM, Burgess CS (2010): Detection of nonnative speaker status from content-masked speech. Speech Commun 52:626-637.

Nilsson M, Soli SD, Gelnett DJ (1996): Development of the Hearing in Noise Test for Children (HINT-C). Los Angeles, House Ear Institute.

Park H (2013): Detecting foreign accent in monosyllables: the role of L1 phonotactics. J Phon 41:78-87.

Sumner M, Kim SK, King E, McGowan KB (2014): The socially weighted encoding of spoken words: a dual-route approach to speech perception. Front Psychol 4:1015.

Van Engen KJ, Peelle JE (2014): Listening effort and accented speech. Front Hum Neurosci 8:577.

Vieru B, Boula de Mareüil P, Adda-Decker M (2011): Characterisation and identification of non-native French accents. Speech Commun 53:292-310. 